Bárbara Martínez ${ }^{1}$

\title{
La muerte como proceso: una perspectiva antropológica
}

\author{
Death as a process: an anthropological perspective
}

\begin{abstract}
This article combines recent approximations that question the homology between death and biological demise from an anthropological perspective with the tradition of studies that review the death rituals in the Andean geographical area. In particular, it examines how in El Cajón, Catamarca, Northeast Argentina the dead are incorporated in the cosmologic scheme, and how death, being the initial step for this to occur, is not merely a biological event but also a highly dynamic social process. Based on ethnographical field work and using multiple-session open interviews and participant observation as methodological tools, it presents an analytical proposal that seeks to transcend the homology between death and biological demise, suggesting a notion that includes not only the organic dimension but also the social dimension: the process of death.
\end{abstract}

Key words Process of death, Absence of vital signs, The coffin
Resumen Este artículo, desde una perspectiva antropológica, combina recientes aproximaciones que cuestionan la homología entre muerte y deceso biológico, con la tradición de estudios que revisan los rituales de muerte en el área geográfica andina. En particular, examina cómo se produce en El Cajón, Catamarca, noroeste argentino, la incorporación de un fallecido al esquema cosmológico, y cómo la muerte, que es un puntapié inicial para ello ocurra, no es meramente un evento biológico sino también un proceso social de gran dinamismo. A partir del trabajo de campo etnográfico, y tomando como herramientas metodológicas entrevistas abiertas de sesiones múltiples y observación participante, presenta una propuesta analítica que procura superar la homología entre muerte y deceso biológico, sugiriendo una noción que incluye la dimensión orgánica, pero también la social: el proceso de la muerte.

Palabras clave Proceso de la muerte, Ausencia de signos vitales, El Cajón
${ }^{1}$ Facultad de Filosofía y Letras, Universidad de Buenos Aires. Puán 430. C1406CQH Buenos Aires Argentina.

bmartinez@filo.uba.ar 


\section{Introducción}

En el esquema mítico de los habitantes de El Cajón, Catamarca, en el noroeste argentino, existen narraciones que identifican una serie de distinciones cosmológicas, que desde una perspectiva analítica son entendidas como "una serie de ideas acerca de la realidad, la cual puede o no aparecer ordenada sistemáticamente en una teoría nativa general". El Cielo es una región donde residen Dios y los santos, que intervienen ante éste en las peticiones de los hombres. De los muertos se sabe que ocupan lugares diversos. Entre ellos, los angelitos, es decir, en términos generales, los niños muertos, ascienden directamente al Cielo gracias a que no han pecado. Los difuntos, personas adultas que vivieron bajo los preceptos de lo moralmente aceptado, pasan un tiempo en el Purgatorio, sitio de ubicación incierta pero del que pueden salir ayudados por las ofrendas y oraciones de los vivos. Los condenados, que son quienes cometieron faltas graves, como suicidio o incesto, siguen un derrotero similar. Las valoraciones sobre estas dos últimas dependen de las interpretaciones de los vivientes sobre la muerte, pero también sobre los sucesos de la vida de los fallecidos, como el adulterio. Para el caso de los condenados, su escape del Purgatorio resulta en extremo infrecuente. Ellos pueden, además, aparecer entre los hombres, enfermándolos e incluso ocasionándoles la muerte.

En este cuadro, la tierra es la morada de los hombres, los animales, las plantas y los seres como la Pachamama. Esta última es una entidad polisémica. Aunque suele presentarse como una anciana con atributos de pastora (huso, sombrero de ala ancha), en ocasiones transmuta en los animales que protege (como los felinos). Es, también, la tierra entendida como sustrato material ${ }^{2}$.

Ahora bien, el deceso de una persona, y su integración en este orden cosmológico, implican una compleja secuencia que obedece al deceso biológico, aunque no se limitan a él.

En este artículo analizo cómo se produce la incorporación de un fallecido al esquema cosmológico, y cómo la muerte, que es un puntapié inicial para ello ocurra, no es meramente un evento biológico sino también un proceso social de gran dinamismo.

Este trabajo combina los resultados de una serie de investigaciones que revisaron los rituales mortuorios en el área geográfica andina ${ }^{3-7}$ con los aportes de los autores que han tratado de superar la aparente homología entre muerte y deceso biológico. Por cierto, esta perspectiva no es nueva. Sabemos que "la muerte no se consuma en un acto instantáneo, sino que implica un proceso lento que en gran número de casos no se da por terminado hasta que la disolución del

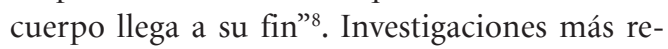
cientes en contextos clínicos han demostrado que lo que se entiende por vivo o muerto depende de las valoraciones de los actores 9 . Incluso los criterios acordados por la biomedicina para declarar formalmente la ausencia de signos vitales se han modificado con la utilización de nuevas tecnologías $^{10,11}$. Además, las dificultades conceptuales para definir la muerte tienen una consecuencia directa en la práctica clínica ${ }^{12}$.

Esta indagación se sitúa en la localidad de El Cajón se sitúa en el Departamento de Santa María, en la provincia de Catamarca (Argentina). Su subsistencia, basada en una economía agrícolo-ganadera, se complementa con el trabajo en plantaciones de frutas, en minería y, más recientemente, con la ayuda que brinda por los Planes de Asistencia otorgados por el estado provincial o nacional. Se encuentra unida a Santa María, el centro político y administrativo del área, por un camino que suele interrumpirse durante la estación de lluvias. La población no cuenta con suministro de energía eléctrica ni posee médicos. Los agentes sanitarios, profesionales de la salud a nivel local, actúan como intermediarios entre los enfermos y el hospital zonal, ubicado en el centro urbano que mencionamos.

En este trabajo, la primera sección especifica la metodología utilizada. Las tres siguientes examinan cómo los rituales transforman a un viviente en un difunto, y cómo esta construcción es social además de biológica. Finalmente la quinta, partiendo de la discusión del material de campo etnográfico, presenta una propuesta analítica que procura superar la homología entre muerte y deceso biológico, sugiriendo una noción que incluye la dimensión orgánica, pero también la social: el proceso de la muerte.

\section{Metodologia}

El análisis que se presenta aquí forma parte de la investigación etnográfica que desarrollo en el área desde el año 2003. Desde el punto de vista metodológico, he realizado entrevistas abiertas y de sesiones múltiples pero, en virtud de las características del tema abordado, la observación participante ha sido una herramienta fundamental. Es que la entrevista, como evento de habla, no 
forma parte de la experiencia comunicativa cajonista, en la que las preguntas directas son consideradas como descortesía ${ }^{13}$. Por ello, mi aprendizaje sobre la vida social local se fundó en mi participación en eventos relevantes y en una convivencia prolongada que me permitió formar parte de sus prácticas cotidianas.

\section{La llegada de la muerte}

\section{El inicio}

En El Cajón, el deceso de una persona puede ser inminente, siguiendo el diagnóstico del agente sanitario. Claro está, también pueden morir repentinamente. Pero a partir de una serie de señales, es posible, en la interpretación local, saber que alguien va a morir.

Los pobladores han reservado un término para designar a los fenómenos que anticipan el deceso: los denominan tapia.

Tapia quiere decir que hay manifestaciones [...] que anuncian la muerte. [...] cuando sucede esto y a la gente dice "Alguien va a morir". Y algunas veces yo he visto que es cierto. Que cuando ha muerto el hombre ese, lo había matado un rayo [...]. (Walter Albarracín, enero de 2004).

Hemos apuntado en otro sitio que, en términos generales, en el contexto local las lógicas clasificatorias y simbólicas suelen tomar su materia prima de la naturaleza ${ }^{14}$. En particular, en relación a la muerte, ciertos animales, actúan como agentes anticipatorios del deceso.

Incluso también la señora del hombre era, es la cocinera de la escuela. Estaba en la cocina, ese día antes, dos días habian aparecido mariposas. O sea, ellos le dicen pirpinto, habian invadido la cocina. $Y$ dice la señora: "¿Qué irá a pasar? ¿Quién irá a morir?". Y yo: "Nooo...”. Al día ha fallecido el marido. (Walter Albarracín, enero de 2004).

En nuestra conversación, Walter entrelazó el evento biológico con la presentación de un tipo específico de mariposas, llamadas pirpinto (Erebus odoratus), revelando indirectamente sugerentes conexiones sobre las que volveremos. Por el momento, nos interesa argumentar que, según surge del trabajo de campo realizado, esta elección no es fortuita. Más precisamente, las asociaciones simbólicas que las personas realizan se nutren de una observación meticulosa y detallada de los hábitos de estos seres.

Teniendo en cuenta la información etnográfica disponible, podemos considerar que, en términos generales, las clasificaciones simbólicas locales trabajan simultáneamente sobre varios ejes, como la continuidad (dando cuenta de cosas que estructuralmente pertenecen a un mismo sistema), la semejanza (posesión común de uno o más caracteres), basándose en caracteres inteligibles (funciones comunes) o en caracteres sensibles (rasgos comunes) ${ }^{15}$. Es posible también que las clasificaciones se organicen a un tiempo combinando uno o más de los puntos que hemos separado a los fines analíticos. Citaremos un ejemplo para cada uno. En el primer caso, entendemos que las víboras y los lagartos pertenecen a una clase de lo considerado nefasto, por eso "mueren" el Viernes Santo, cuando Cristo ha muerto y "nacen" el 1 de agosto, días que también son nefastos. En algunos momentos significativos del ciclo ritual anual, como la Semana Santa, su matanza representa, además, el triunfo de los hombres sobre las fuerzas que capturaron y crucificaron a Cristo. Se trata de animales polisémicos que en algunas acciones rituales significativas, como las que se llevan a cabo en ese lapso, también sustituyen a los judeos, figuras míticas que gracias a la incesante la prédica religiosa, los cajonistos han asimilado con los sicarios de Cristo.

Como relación al criterio basado en la semejanza, hemos advertido, siguiendo la exégesis nativa, que ciertos cabritos negros son oscuros y ostentan cuernos, como el maligno que, en términos muy generales, suele estar identificado con el demonio. Pueden citarse como casos de clasificación basados en su función, el sanaloto (nido de un minúsculo insecto que crece en cuevas y abrigos rocosos y que la autora se encuentra en proceso de identificación de su nombre científico), y la chachacoma (senecio graveolens, generalmente utilizada en la medicina local por sus propiedades sedantes y su efectividad contra las enfermedades respiratorias), pues juntándolos sirven para sahumar durante el primero de agosto. Por fin, el alga "cola de sapo" que crece en la orilla de los ríos, recuerda la cola que el sapo pierde en su pasaje de larva a adulto.

El sistema simbólico local implica el uso de lógicas clasificatorias que expresan metáforas de los procesos que conciernen al mundo social humano. Pero además, en el caso de la muerte, los animales aparecen activamente en las formulaciones, donde ellos mismos surgen como atributos $^{16}$. Por ello, a nuestro entender, la elección de los animales vinculados con la muerte, el morir, la putrefacción, lo inmundo o lo nefasto, descansa con frecuencia en las dinámicas de sus procesos biológicos. En el Cuadro 1 hemos esquematizado algunos ejemplos de animales, sus patrones de conducta y los procesos sociales con los que los pobladores los han asociado. 
Cuadro 1. Asociaciones simbólicas de animales, sus conductas y hábitos.

\begin{tabular}{|l|l|l|}
\hline \multicolumn{1}{|c|}{ Animal } & \multicolumn{1}{|c|}{ Conducta/hábitos } & \multicolumn{1}{c|}{ Asociaciones simbólicas } \\
\hline Carancho (Caracara plancus) & Carroñero. Plumaje oscuro. & Anticipación de la muerte \\
\hline Pirpinto (Erebus odoratus) & Mariposa nocturna. & Anticipación de la muerte \\
\hline Cóndor (Vultur gryphus) & $\begin{array}{l}\text { Longevidad. Habita altas } \\
\text { cumbres y peñascos }\end{array}$ & $\begin{array}{l}\text { Longevidad/inmortalidad. Vinculaciones con } \\
\text { el cielo donde habitan Dios y los santos. }\end{array}$ \\
\hline
\end{tabular}

En el primer caso, el carancho es un ave que se alimenta de los restos de los animales muertos que encuentra a su paso. De plumaje negruzco en algunos sectores de su cabeza, alas y torso, ocasionalmente se convierte en un cazador oportunista que atrapa animales jóvenes o heridos, lastimando primero sus ojos, aumentando así su indefensión y causando paulatinamente el deceso. Además, emite un sonido punzante muy característico. En El Cajón la presencia de esta especie es considerada un presagio inequívoco de la muerte de un poblador. "Cuando aparece el carancho, es seguro (que alguien morirá)" escuché varias veces decir allí. Como mencionamos, los cajonistos han sintetizado equivalencias estructurales que articulan la sociedad y la naturaleza, donde los animales actúan como metáforas de los procesos humanos. En el caso del carancho, su plumaje oscuro evoca las asociaciones muerte/interrupción del ciclo vital/luto. Es que en el terreno del simbolismo cromático, el color negro está vedado a aquellos momentos de la vida social que tienen como objeto la propiciación. Así, las flores confeccionadas con lanas de colores que los hombres colocan en el ganado durante las señaladas, que son rituales donde, en sentido amplio, se promueve la fertilidad, no deben ser negras, pues esto ocasionaría la muerte de los ejemplares y la consiguiente disminución del rebaño. Volviendo a nuestro ejemplo, debemos agregar también que la materia de la que se alimenta el carancho es fétida, como lo son los procesos biológicos postmortuorios.

Volvamos a nuestro ejemplo inicial. El pirpinto es una mariposa oscura, de gran tamaño y de hábitos nocturnos. Su aparición igualmente suele ser interpretada en términos de anticipación del deceso de un poblador, tal como lo recordaba Walter. La condensación simbólica, en este caso, se nutriría de la idea de que la noche es un dominio cosmológico que reúne a los pirpintos y a los muertos ${ }^{17}$.
Pero además de estas metáforas clasificatorias, existen otros modos de tapia. A nivel local, hemos revisado distintas acepciones para el término alma $^{18}$. Nos interesa aquí aquella que ilustra que ésta, con anterioridad a la constatación de la ausencia de signos vitales, puede desprenderse del cuerpo y manifestarse mediante acontecimientos infrecuentes.

Escuchan el golpe a la ventana, o se escucha pasos, o en la escuela, por ejemplo, uno de los maestros había escuchado que le golpeaban la puerta o se sentían pasos y al poco tiempo ha fallecido un ex docente de la escuela. Una vez veníamos bajando de noche nosotros [...] habíamos bajado el cerro ese. La mula se... la habíamos atado ya se habíamos bajado para caminar un poco ya veníamos cansados y empieza a bufar la mula. Ya ha empezado a quererse disparar la mula y dice el baqueano: -Uh! Quien irá a morir! - ¿Por qué? - Le digo Debe andar un alma por ahí, deambulando. - Pero como íbamos ya como las doce de la noche... Al otro día nos enteramos que había fallecido un hombre por ahí cerca. [...] Anda un alma que le llaman. De la persona que va a fallecer. (Walter Albarracín, enero de 2004)

Esta vez, en su explicación, Walter indirectamente revela una cuestión central a nuestro análisis, pues describe que, en términos ontológicos, una dimensión del ser, el alma, se despliega de forma independiente del cuerpo, anticipando el evento biológico inminente. Entonces, aunque la persona (cuya identidad no se conoce aún) está clínicamente viva, el proceso social por el cual la gente de El Cajón simboliza una nueva muerte, ya se ha iniciado.

Citaremos otro ejemplo que sintetiza los argumentos que esbozamos. En marzo de 2010, Sabino, un poblador muy prestigioso en virtud de sus facultades como cazador, desapareció de su casa durante días. Desde ese momento, y hasta el hallazgo de su cadáver, al pie de una peña, la población se encontraba virtualmente aterrori- 
zada. A través de ciertos oráculos locales, como la lectura de la llama de la vela, y el comportamiento de algunos animales (como el sobrevuelo de caranchos), se sabía que el hombre había fallecido. Su alma estaba hace días vagando entre los vivos, apareciéndoseles en sueños y mediante extraños ruidos. De modo que, con el correr de los días, sólo restaba hallar su cadáver. Sabino, aún antes de la confirmación fehaciente del deceso biológico, y aunque existieran algunos rumores que lo implicaban en una supuesta huida hacia otros territorios, para la gente local ya se encontraba muerto.

\section{Velorio, novena y lavatorio}

Una vez constatada la ausencia de signos vitales, las reglas que rigen los intercambios recíprocos promueven que buena parte de los pobladores se acerque a colaborar con la familia afectada. Esencialmente se ocupan de vestir al difunto, cocinar los alimentos que se sirven en un banquete ritual extendido a los participantes, organizar los rezos, elaborar su túnica, realizada a semejanza de aquella utilizada por Cristo y los sacerdotes, y ocuparse de obtener un ataúd en la ciudad. La gente acompaña al muerto y a sus parientes más próximos rezando y bebiendo durante toda la noche. El don cobra en este caso forma de trabajo. Este tipo de intercambio se realiza en razón de la relación social que une a las partes, volviéndose más obligatoria entre parientes $^{19}$. En el contexto ritual como el que aquí nos ocupa, la distancia sociológica hace que la obligatoriedad sea menos acentuada a medida que nos alejamos en grados de parentesco del grupo nuclear de la familia que, como forma ideal, está compuesta por un hombre, la mujer y los hijos de ambos, quienes comparten la misma vivienda. Cuando otra familia se encuentra en la misma situación, se espera que los parientes ayudados esta vez, retribuyan el trabajo.

El velorio se desarrolla durante aproximadamente un día. Si el fallecido es un adulto, el cuerpo se coloca extendido en una cama, rodeado de cuatro velas en forma de cruz, en general en un cuarto separado de la casa, dispuesto según las comodidades que permiten la libre circulación de las personas, más que siguiendo coordenadas ligadas al simbolismo cardinal. Los niños, en cambio, son velados sobre una mesa. Habiendo preparado grandes cantidades de comida y ofrecido bebida en abundancia, los parientes identifican la puerta donde transcurre el velorio con un paño negro al que se ha cosido una cruz blanca.
La muerte de un sujeto imprime un nuevo conjunto de reglas que afectan tanto a él como a la totalidad de la sociedad. Pero además, su estado es concebido como un itinerario que implica una inversión de las acciones a las que estuvo sujeto durante su vida, transitando un camino hacia una nueva estructura cosmológica y ontológica. Para ello se requiere de la incorporación de hábitos que le impondrán una distinción profunda con los anteriores y un reacomodamiento a nuevas coordenadas espaciales. Es que el mundo de los muertos es, pues, inverso en muchos sentidos al de los vivientes. De algún modo, es como si los senderos que los hombres transitan en el pastoreo y las actividades de la vida cotidiana se subvirtieran con el deceso. Por eso, cuando el velorio concluye, varios hombres cargan el ataúd y recorren los cuatro rincones de la casa. Recapitulando las coordenadas que rigen el orden cosmológico, el cadáver transita la vivienda que él mismo alguna vez edificó en el proceso social de construir una nueva familia. En sentido inverso a la trayectoria de su vida, el cadáver comienza a desandar el mundo que ahora pertenece al dominio de los vivos. Es que, de acuerdo a la subversión de las coordenadas espaciales que atañen al nuevo fallecido, durante las jornadas previas al deceso biológico, el alma recorre los espacios conocidos. En ese proceso, persiguiendo el ideal del cuerpo íntegro, el alma recupera los retazos personales como las uñas y el pelo recortados durante la vida.

Volviendo el velorio, por regla general, los familiares deben evitar trasladar el cuerpo, pues se cree que si lo hicieran, también podrían fallecer, ya que el difunto regresaría para llevarlos consigo. Es una señal de ello que el cajón sea más pesado de lo habitual.

Las actividades cotidianas, como el dictado de clases en la escuela local, se suspenden, y gran parte de los pobladores acuden al entierro. El camino hasta el cementerio se encuentra plagado de prescripciones rituales. Los descansos, las ofrendas, las libaciones y las oraciones son ineludibles. Los condenados pueden dificultar el camino de los dolientes, enfermándolos.

Finalmente, el ataúd es depositado en una fosa y sobre él se arrojan puñados de tierra, cigarrillos, alcohol y una ración de hojas de coca. Si durante su vida una persona exigió ofrendas especiales para este momento, los parientes deben recordarlas y efectuarlas, de lo contrario el muerto podría reclamarlas en sueños. La obligación de dar allana el camino para los intercambios equilibrados y obstruye la posibilidad de que se 
produzcan vínculos de reciprocidad negativa.

La jornada del deceso biológico marca el inicio de una serie de rezos que durará nueve días, llamada novena. La acción ritual continúa con un nuevo velorio, pero esta vez el conjunto de pertenencias del fallecido emulará la presencia del cadáver. Análogamente al momento en que el cuerpo estaba presente, los familiares ofrecen un banquete ritual extendido, los rezos continúan durante la noche y las personas reciprocan con trabajo. Largas y monótonas, las letanías ponen en contacto al muerto con los santos, seres que también han sido en un tiempo como los hombres, y que hoy, cumpliendo con las trayectorias de los intercambios, interceden por ellos.

Más tarde, el lavatorio implica la limpieza de cada una de las prendas de vestir, los objetos personales y herramientas que componen el bulto de pertenencias velado. Como mencionamos, en el tránsito al mundo de los muertos, el rumbo se invierte y con él cada fragmento de la vida social anterior. Así, por ejemplo, un no pariente o una persona ligada al difunto desde el tercer grado de parentesco (pues a los más cercanos les está vedado) lava, desovilla y vuelve a ovillar los atados de lana que pertenecieron al difunto, interiorizando las nuevas coordenadas cosmológicas y ontológicas a las que deberá ajustarse el fallecido (Figura 1).

\section{Trayectoria postmortuoria}

En el esquema de los intercambios, la propiciación del sendero postmortuorio cabe también al vínculo entre los animales y los hombres. En las tareas de pastoreo, las personas suelen contar con

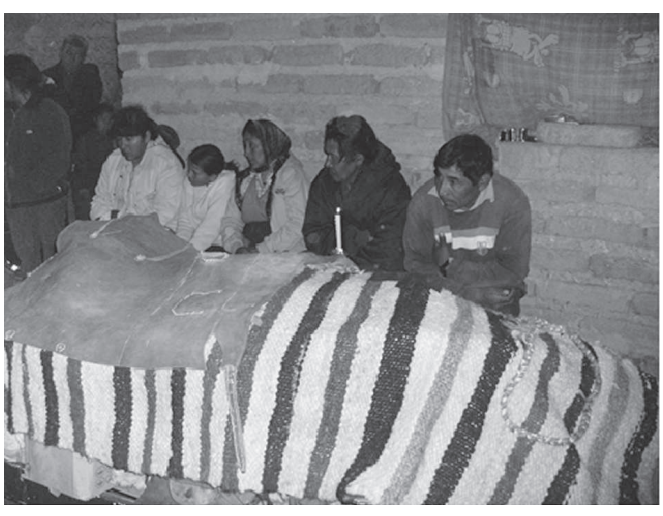

Figura 1. Velatorio de las posesiones de Sabino. Sobre el puyo pueden observarse la honda y la montura. varios perros que cumplen funciones de arreo y protección. Como en el tránsito de la vida, por el sendero de los muertos el hombre no viaja solo. La casa se convierte en el escenario de sacrificio de uno de los perros del difunto, idealmente negro, pero también el más cercano e inseparable. Es que, a nivel local, los perros negros son intermediarios vinculados con uno y otro mundo, diestros en vérselas con las fronteras que obstaculizan a los hombres. Durante la vida del dueño están ligados muy especialmente a su protección frente a los seres nocturnos, como el maligno y los muertos. Entonces, muerto y enterrado con una chuspaes decir, una pequeña bolsa tejida que los pastores utilizan cotidianamente -, alimentos, bebida y una ración de coca, se le encomienda la tarea de que sirva de guía al alma por la nueva geografía. Sinuoso, riesgoso y levemente fortuito, el tránsito por los senderos de la topografía mítica postmortem demanda la asistencia de este aliado cotidiano de los hombres. El ritual motiva nuevas ecuaciones. Las transferencias metafóricas se revelan transparentes en este punto. Las cualidades del perro-guía en el mundo de los vivos se desplazan a un nuevo plano cosmológico, convirtiéndolo en un asistente de la trayectoria postmortuoria.

La nueva y desconocida geografía con la que el alma se enfrenta se organiza con fidelidad, siguiendo el modelo del paisaje que rodea al pueblo. Los cajonistos creen que el alma deberá atravesar tres corrientes: un río de agua, un río de leche y un río de sangre.

El primero, se sabe, surgió como resultado de las lágrimas que lloran las madres por sus hijos muertos. Una tarde en que pastábamos sus cabras, Doña Telma, una amable anciana, me ilustró sobre este punto. Algunas madres, explicó, no se entristecen por la muerte de sus hijos. Pero otras sí, como la virgen cuando martillaron el cuerpo de Cristo a la cruz.

Cuando pregunté sobre el significado del segundo río, las personas vagamente respondieron que lo ignoraban. La exégesis nativa se interrumpe en este punto.

Por fin, el tercer curso está integrado por la sangre de los fallecidos por laceraciones y graves lesiones corporales. Esta es la corriente más difícil de sortear, y en la que más se necesita del perro-guía. Alimentado por las provisiones que lleva en la chuspa y montado en su lomo, el alma emprende la penosa tarea de atravesar este curso, para alcanzar un sitio de nombre y ubicación incierto, del otro lado del río.

Vemos que el agua (o las lágrimas en la interpretación cajonista) y la sangre describen aspec- 
tos fisiológicos, emanaciones corporales. Podemos sugerir como hipótesis que la leche, como los anteriores, también remite a aspectos biológicos, posiblemente vinculados con la maternidad (como el dolor materno que expresan las lágrimas), la reproducción y la vida que se está dejando atrás.

Pero en otras versiones, el alma sólo atraviesa una corriente, el río Jordán. En marzo de 2007 realicé una entrevista a Adelino, un párroco que por entonces vivía en Santa María y era el encargado brindar asistencia espiritual a la gente de El Cajón. Interrogado sobre el simbolismo católico del Río Jordán, brindó una sugerente respuesta:

La gente mezcla las cosas y, en realidad, todo esta lleno de significados y simbolismos. El bautismo es, también, un identificar, un introducirse, diríamos, en las aguas, en la muerte a los vicios de este mundo, y a los vicios y a las maldades de este mundo, para salir limpios y bautizados. Porque eso es el bautismo, la limpieza del pecado original y de los pecados personales y comenzar desde cero, una vida nueva. Entonces, Jesús, en realidad, él también, se sumergió en las aguas del Jordán y allí se dejó bautizar por Juan el Bautista. Pasó como pecador sin ser pecador, porque era el santo y era el hijo de Dios y el hijo de Dios no tiene pecados. Pero quiso aparecer como el pecador, él, y para darnos ejemplo y decirnos que hay que sumergirse en las aguas, limpiarse, para comenzar una vida nueva. (Padre Adelino, marzo de 2007).

Es posible, entonces, que el río Jordán actúe entre los cajonistos como una metáfora que, además de condensar el cruce entre una vida y la siguiente, de cuenta de modificaciones ontológicas entre un estado y otro (vg. impureza terrenal vs. pureza ulterior, etc.). Fragmentos de la acción ritual tendientes a la purificación del muerto, como la asimilación de sus ropas a las de Cristo y a las del sacerdote - pues es vestido con una túnica y un cordón que emula la vestimenta de ambos - apoyan esta interpretación.

A partir de este tránsito postmortuorio, una nueva trayectoria de intercambios articula al fallecido con los vivientes. Integrado a una condición ontológica diferente, está en situación de interceder ante Dios y los santos, a favor de los vivientes. La dinámica de la reciprocidad toma un nuevo rumbo, y se acentuará en su retorno, durante las celebraciones del 1 y 2 de noviembre, lapso en que, sintéticamente, se cree que los difuntos vuelven a visitar a los hombres. Estos los colman con una serie de ofrendas, cumplimentando con las formas de intercambio recíproco que los une ${ }^{20}$.
Finalmente, el primer aniversario de la muerte marca el momento en el que los sucesores reciben en herencia los bienes del difunto (como por ejemplo el ganado). Es a partir de entonces que se ha finalizado el conjunto de la acción ritual.

\section{La muerte como proceso}

Las etapas del ritual que repasamos, que conducen a la transformación de un poblador en un difunto, nos retrotraen a la discusión inicial sobre la relación entre la conceptualización de muerte, y sus articulaciones con el deceso biológico. Mientras que, en el último tiempo, la literatura antropológica se ha preguntado qué se entiende por el inicio y finalización de la vida ${ }^{21}$, la cuestión del deceso biológico como construcción social también ha cobrado importancia ${ }^{22}$. Asimismo, las definiciones de los actores sobre la muerte han sido recientemente revisadas. Se ha sugerido que la muerte social ocurre cuando una persona, esté biológicamente muerta o no, ha dejado de ser tratada por el resto como un agente activo dentro del entramado de relaciones sociales ${ }^{23}$. De acuerdo con esta definición, una persona puede estar socialmente muerta, aunque desde una perspectiva biológica se encuentre viva. Ejemplos etnográficos que acreditan estos procesos ${ }^{24}$. Incluso en mi propio trabajo, he apuntado el caso de María, una joven de El Cajón, que en un contexto de embarazo adolescente y acusaciones de incesto, fue invisibilizada por el resto del grupo. El suicidio de la joven culminó con un proceso de muerte social iniciado tiempo antes ${ }^{25}$.

De modo similar a la idea de muerte social, en este trabajo hemos revisado cómo la sociedad cajonista, a partir de una serie de eventos anticipatorios, actúa ante un eminente y aún no corroborado deceso biológico, de modo muy cercano a como lo haría si éste ya se hubiese concretado. De este modo, la interpretación social de la muerte no siempre coincide con un evento orgánico.

Pero también, en el contexto estudiado, aún cuando el deceso biológico ocurre, la muerte no se limita a ese evento puntual, sino a una amplia serie de acciones rituales que hacen que un poblador deje de formar parte del conjunto de los vivos, para adquirir una condición ontológica y cosmológica diferente. Es a partir de esto, y del cumplimiento de las obligaciones recíprocas que lo unen con los vivos, que se consolida su posición estructural como difunto.

Por ello, proponemos llamar proceso de la muerte al período que comienza con los eventos de anticipación del deceso hasta la expulsión del 
difunto luego de los ritos que se celebran en el primer aniversario. Esta categoría analítica permite resaltar que la muerte no se restringe a un evento biológico único, sino a una serie de sucesos y contingencias que son socialmente interpretados como relevantes. Esta idea, además, tiene la ventaja de correr el foco de atención de la variable orgánica, y de las dificultades que los profesionales de la biomedicina han señalado para el establecimiento del momento concreto del deceso.

\section{Conclusiones}

Como adelantamos, es posible identificar a la muerte a partir de un evento biológico, pero también de una amplia serie de eventos anticipatorios, que toman su materia prima de la naturaleza (como la presencia de animales) o implican el "desprendimiento" de partes que componen la ontología cajonista (como el alma), entre otros. A ello se suma un conjunto de acciones rituales (entre las que señalamos el velorio, el entierro, el lavatorio, la travesía posmortuoria y la distribución de algunos bienes al cabo de un año) que suponen la interacción dinámica de elementos que constituyen las formas mediante las que socialmente se transforma un viviente en un difunto. Todo ello conforma un proceso continuo, que en el texto se ha separado sólo a los fines analíticos.

Este devenir de un nuevo fallecido, a su vez, está intrincablemente relacionado con las concepciones de la ontología y cosmología local. Ello se verifica en transformaciones como el "separación" del alma, pero también en su paulatina incorporación al plano celeste. A su vez, ello está marcado por la inversión de las trayectorias que caracterizan al mundo de los vivos, llamada desandar.

Desde un punto de vista general, la concreción de estos rituales expresa las alianzas recíprocas y refleja las tensiones al interior del grupo. En un plano más abstracto, es a partir de ellos que los nuevos difuntos se incorporan al plano cosmológico, actuado como intermediarios entre los hombres, Dios y los santos, y promoviendo la fertilidad y la abundancia. Esto se verifica en las peticiones a lo largo de todo el año, que se incrementan cuando se intensifican los intercambios, durante el Día de Todos los Santos y el de las Almas.

Dentro de este contexto sociorreligioso, las valoraciones de los personas, sustentadas en la trayectorias de la vida, y en ocasiones sobre el modo en que se produce el deceso biológico, definen categorías específicas de fallecidos, como los angelitos, los condenados y los difuntos. Es también a partir de ellas que esporádicamente algunos pobladores pueden ser socialmente invisibilizados, experimentando un tipo de muerte no biológica, que hemos definido como muerte social.

Más allá de estas evaluaciones al interior de la sociedad local, la incorporación de un fallecido a partir del conjunto de eventos que denominamos proceso de la muerte, no necesariamente implica una homología entre deceso biológico y muerte. Como apuntamos, la trayectoria del alma cajonista involucra una serie de pasos, que comienzan previamente. Según mencionamos, el alma abandona el cuerpo días antes, y permanece vagando entre los vivos durante el velorio $y$ entierro. Luego del deceso biológico, el velorio, el velorio de las pertenencias y el lavatorio intensifican la separación del mundo de los vivos. El sacrificio de su perro marca el momento en que el alma inicia el trayecto por la topografía postmortem junto a su compañero, hasta llegar a un lugar incierto, luego de atravesar los ríos de agua, de leche y de sangre en algunas versiones, o bien el río Jordán, en otras. De modo que tanto los anteriores como los rituales de repartición de bienes al cabo de un año, concretizan la nueva posición estructural del difunto.

Aunque en otro sitio nos hemos ocupado de las trayectorias de acercamiento de los cajonistos a la biomedicina ${ }^{26}$, queda pendiente indagar situaciones concretas de interpretación de la muerte en el contexto hospitalario, cuando se realizan derivaciones hacia el Hospital de Santa María. Estos interrogantes, que será necesario contestar más adelante, permitirán revisar cómo los cajonistos definen el deceso en otros contextos geográficamente situados. 


\section{Referencias}

1. Wright P. Ser $\sim$ en $\sim$ el $\sim$ sueño. Crónicas de historia y vida toba. Buenos Aires: Biblos; 2008.

2. Pallet A. Introducción a la sociología de la religión en el contexto andino. En: Spedding Pallet A, compiladora. Gracias a Dios y a los achachilas: ensayos de sociología de la religión en los Andes. La Paz: ISEAT, Plural editores; 2004. p. 11-71.

3. Harris O. Los muertos y los diablos entre los laymi de Bolivia. Chungara 1983; 11:135-152.

4. De Hoyos M. Saliendo el cajón por el Río Jordán: costumbres funerarias en el Valle del Cajón. Chungara 2001; 15(2):249-252.

5. Van Kessel J. El ritual mortuorio de los aymara de Tarapacá como vivencia y crianza de la vida. Chungara 2001; 33(2):221-234.

6. Fernández Juárez G. Apxatas de difuntos en el altiplano aymara de Bolivia. Rev Esp Antropol Am 2006; 6(1):165-182.

7. Azevedo VR. Miroirs de l'autre vie. Pratiques rituelles et discours sur les morts dans les Andes de Cuzco (Pérou). Nanterre: Société d'Ethnologie; 2008.

8. Hertz R. La muerte. La mano derecha. México: Alianza Editorial; 1990.

9. Sudnow D. La organización social de la muerte. Buenos Aires: Tiempo contemporáneo; 1971.

10. Lock M. Death in technological time: locating the end of meaningful life. Med Anthropol Q. 2002; 10(4):575-600.

11. Uzal L. El cuerpo y lo muerto. El cadáver, la identidad $y$ las intervenciones sobre los cuerpos en la práctica funeraria [tesis]. Universidad de Buenos Aires: Facultad de Filosofía y Letras; 2013.

12. Siqueira-Batista R, Schramm FR. Eutanásia: pelas veredas da morte e da autonomía. Cien Saude Colet 2004; 9(Supl. 1):31-41.

13. Briggs C. Learning how to ask. A sociolinguistic appraisal of the role of the interview in social science research. Cambridge: Cambridge University Press; 1986.

14. Martínez B. Ríos de agua, ríos de leche y ríos de sangre. Travesías cosmológicas del alma cajonista [tesis]. Universidad de Buenos Aires: Facultad de Filosofía y Letras; 2011.

15. Lévi-Strauss C. El pensamiento salvaje. México: Fondo de Cultura Económica; 1997.

16. Urton G. Animal myths and metaphors in South America. Salt Lake City: University of Utah Press; 1985.
17. Martínez B. Ríos de agua, ríos de leche y ríos de sangre. Travesías cosmológicas del alma cajonista [tesis]. Buenos Aires: Universidad de Buenos Aires; 2011.

18. Martínez B. Ríos de agua, ríos de leche y ríos de sangre. Travesías cosmológicas del alma cajonista [tesis]. Buenos Aires: Universidad de Buenos Aires; 2011.

19. Mayer E. Las reglas del juego en la reciprocidad andina. En: Alberti G, Mayer E, compiladores. Reciprocidad e intercambio en los Andes peruanos. Lima: Instituto de Estudios Peruanos; 1974. p. 37-65.

20. Martínez B. Rituales de muerte en el sector sur de los Valles Calchaquíes. En: Hidalgo C, compilador. Etnografías de la muerte. Rituales, desapariciones, VIH/SIDA y resignificación de la vida. Buenos Aires: Clacso, Ciccus; 2010. p. 87-108.

21. Kaufman SR, Morgan LM. The Anthropology of the beginnings and ends of life. Annu Rev Anthropol 2005; 34(1):317-341.

22. Fratezi FR, Ozello Gutierrez BA. Cuidador familiar do idoso em cuidados paliativos: o processo de morrer no domicílio. Cien Saude Colet 2011; 16(7): 3241-3248.

23. Mulkay M. Social death in Britain. In: Clarke D, editor. The Sociology of Death. Oxford: Blackwell; 1993. p. 31-50.

24. Hallam E, Hockey J, Howarth G. Beyond the body. Death and social identity. London: Routledge; 1999.

25. Martínez B. Ríos de agua, ríos de leche y ríos de sangre. Travesías cosmológicas del alma cajonista [tesis]. Buenos Aires: Universidad de Buenos Aires; 2011.

26. Martínez B. Sistema biomédico y medicina "tradicional" en El Cajón, Catamarca, Noroeste Argentino. En: Soares LVR, compiladora. Pobreza, desigualdad y salud. Buenos Aires: Ediciones Clacso; 2013.

Artigo apresentado em 30/04/2013

Aprovado em 22/05/2013

Versão final apresentada em 06/06/2013 